\title{
Optimization of Microspeaker Diaphragm Pattern Using Combined Finite Element-Lumped Parameter Models
}

\author{
Mingsain R. Bai, Ching-Yu Liu, and Rong-Liang Chen \\ Department of Mechanical Engineering, National Chiao-Tung University, Hsin-Chu 300, Taiwan
}

\begin{abstract}
Microspeakers in portable devices are expected to meet a number of conflicting requirements such as miniaturization, high output level, and good sound quality. In contrast to large loudspeakers, the structures of microspeakers are generally simplified by removal of the suspension. The diaphragm serves as not only a sound radiator but also the suspension. Thus, the pattern design of the diaphragm is crucial to the overall response and performance of a microspeaker. The traditional approach for modeling microspeakers using lumped parameter models is generally incapable of modeling flexural modes in high frequencies. In this paper, we present a hybrid approach that combines finite-element analysis (FEA) and electro-mechano- acoustical (EMA) analogous circuit to provide a more accurate model than the conventional approaches. In particular, the minute details of diaphragms are taken into account in calculating the mechanical impedance of the diaphragm-voice coil assembly using FEA. The mechanical impedance is then incorporated into the lumped parameter model. The responses can be simulated by solving the loop equations. On the basis of this simulation model, we optimize the pattern design of the diaphragm using the Taguchi method. In addition, we determine the optimal number of diaphragm corrugations by using sensitivity analysis.
\end{abstract}

Index Terms-Diaphragm pattern optimization, electroacoustic analogous circuits, lumped parameter models, voice-coil microspeaker finite-element analysis.

\section{INTRODUCTION}

M INIATURIZATION is the trend of the $3 \mathrm{C}$ (computer, communication, and consumer electronics) portable devices such as mobile phones, personal digital assistants (PDAs), and MP3 players. Loudspeakers in these devices are required to play speech and music signals with acceptable loudness and sound quality. This demand poses a difficult problem to the design of microspeakers whose physical sizes are usually very small. In contrast to large loudspeakers, the structures of microspeakers are generally simplified enough with suspension removed. The diaphragm serves as not only a sound radiator but also the suspension. Thus, the pattern design of the diaphragm is crucial to the overall response and performance of a microspeaker. In this paper, a hybrid approach that combines finite-element analysis (FEA) and electro-mechano- acoustical (EMA) analogous circuit is presented to provide a more accurate model than the conventional approaches. In particular, the minute details of diaphragms are taken into account in calculating the mechanical impedance of the diaphragm-voice coil assembly using the FEA. In order to meet the requirement of output level and sound quality delivered by microspeakers, an optimization procedure is also presented to determine the optimal pattern and dimensions of the diaphragm.

Traditionally, most loudspeaker studies focused on large loudspeakers. In recent years, however, microspeakers have received increased research attention owing to rapid development of $3 \mathrm{C}$ industries. The characteristics of microspeakers have been studied extensively in a variety of aspects, including the

Digital Object Identifier 10.1109/TMAG.2008.923316

Color versions of one or more of the figures in this paper are available online at http://ieeexplore.ieee.org. structure dynamics of the diaphragm, the voice-coil impedance properties of cover perforation [1]-[4], electronic compensation [5], and structural optimization [6]. A well-known method to model of dynamic moving-coil microspeakers is through the use of the EMA analogy. Lumped parameter models can be established, with the aid of such approach [7]-[9]. Thiele and Small (T-S) parameters of the microspeaker need to be experimentally identified prior to the response simulation [10]. Using the analogous circuit, the dynamic responses of microspeaker can readily be simulated, enabling the ensuing design [11].

Despite the simplicity, the lumped parameter model is incapable of predicting the higher-frequency response of the microspeaker which is strongly influenced by the diaphragm, corrugation, and the enclosure. Modeling of the flexural motion of the diaphragm calls for more sophisticated techniques such as FEA. Natural frequencies and mode shapes of the diaphragm-voice coil assembly can be calculated by FEA [12]-[14]. Kwon and Hwang [15] used FEA to examine acoustic performance of microspeakers in lower frequency region for various designs of diaphragm. Chao et al. [16] modeled a microspeaker with a corrugated diaphragm using FEA. The electromagnetic, mechanical, and acoustical subsystems of the speaker were represented by a coupled FEA model. The response of three corrugation angles of $15^{\circ}, 45^{\circ}$, and $75^{\circ}$ were investigated. FEA is employed in this paper to model the diaphragm of microspeaker. However, a feature of this work that differs itself from the previous studies is that the FEA model of the diaphragm is modified into a lumped parameter model, where mechanical impedance of the diaphragm-voice coil assembly is obtained using harmonic analysis of the FEA model. This facilitates tremendously the coupling of the diaphragm model with the models of the rest of the system such as an acoustical enclosure that is usually represented by a lumped parameter model. Using this simulation platform, the voice-coil impedance and the on-axis sound pressure level (SPL) of the 
microspeaker can be calculated by solving the loop equations [17] of the coupled EMA analogous circuit.

Another feature of the paper is that the aforementioned hybrid FEA-lumped parameter model is introduced to extend the design optimization developed in [6]. The diaphragm pattern is optimized using the Taguchi method [18], [19]. In addition, the optimal number of diaphragm corrugation is determined via sensitivity analysis. A number of performance measures concerning the lower cutoff frequency $\left(f_{0}\right)$, the upper cutoff frequency $\left(f_{1}\right)$, mean SPL in the piston range, and the flatness of SPL response are weighted and summed to constitute the cost function. Using the best result of the Taguchi analysis as initial condition, sensitivity analysis is then carried out to determine the number of corrugations for the diaphragm. The thus found optimal design will be compared with the nonoptimal design in the paper.

\section{MODEL OF MiCROSPEAKERS}

Traditionally, lumped parameters methods with EMA analogy are commonly exploited to model loudspeakers. Despite the simplicity, the conventional methods are applicable to model the dynamics in low-frequency regime, especially in the neighborhood of fundamental resonance. However, this may not be sufficient for microspeaker analysis. The lumped parameter model can not predict well the high frequency responses of microspeakers that may play an important role in overall performance such as output level and roll-off frequency. In this work, the diaphragm-coil assembly of microspeaker will be modeled using FEA. The FEA model will be combined with the EMA analogous circuit to establish a fully coupled model for the microspeaker.

\section{A. EMA Analogous Circuit of Microspeaker}

A sample of moving-coil microspeaker with a $16.4 \mathrm{~mm}$ diameter and $4.3 \mathrm{~mm}$ thickness is shown in Fig. 1. The front and rear view of the microspeaker are shown in Fig. 1(a) and (b), respectively. The EMA analogous circuit of this microspeaker can be established in Fig. 2. The coupling of the electrical domain and the mechanical domain is modeled by a gyrator, whereas the coupling of the mechanical domain and the acoustical domain is modeled by a transformer [9]. The T-S parameters can be identified via electrical impedance measurement [9] and [10], as summarized in Table I. The dynamic response of the microspeaker can be simulated on the platform of this model.

\section{B. Finite-Element Analysis of the Diaphragm-Voice Coil Assembly}

The FEA is applied to model the diaphragm-voice coil assembly shown in Fig. 3(a)-(b) with dimensions summarized in Table II. The material properties of the diaphragm-voice coil assembly are included in Table III. The FEA is conducted using ANSYS [20], where the element "shell 63" is used. The shell element has four nodes and 6 degrees of freedom $\left(U_{x}, U_{y}, U_{z}, \mathrm{ROT}_{x}, \mathrm{ROT}_{y}, \mathrm{ROT}_{z}\right)$ at each node. The finite-element model and the mesh of the diaphragm with voice-coil are shown in Fig. 4(a)-(b). The boundary conditions are selected that all degrees of freedom for the outer rim of the

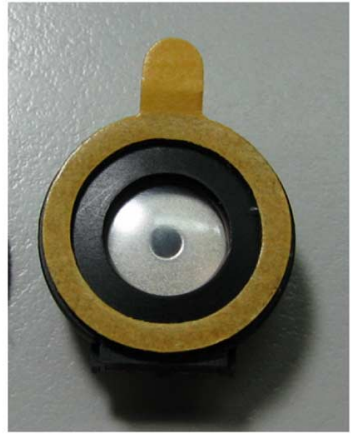

(a)

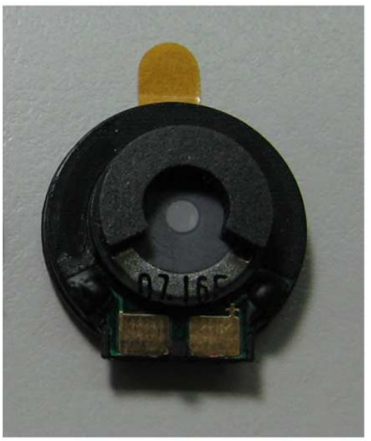

(b)

Fig. 1. Photos of a mobile phone microspeaker. (a) Front view. (b) Rear view.

TABLE I

EXPERIMENTALLY IDENTIFIED LUMPED PARAMETERS OF A MICROSPEAKER

\begin{tabular}{cccc}
\hline \hline Parameters & Value & Parameters & Value \\
\hline$a$ & $6.20 \mathrm{~mm}$ & $B l$ & $0.54 \mathrm{~T} . \mathrm{m}$ \\
$f_{0}$ & $791.9 \mathrm{~Hz}$ & $C_{A S}$ & $1.55 \mathrm{e}-11 \mathrm{~m}^{5} / \mathrm{N}$ \\
$R_{E}$ & $7.76 \mathrm{ohm}$ & $M_{A S}$ & $2598.07 \mathrm{~kg} / \mathrm{m}^{4}$ \\
$R_{E S}$ & $4.40 \mathrm{ohm}$ & $R_{A S}$ & $4.52 \mathrm{e} 6 \mathrm{~N} . \mathrm{s} / \mathrm{m}^{5}$ \\
$Q_{M S}$ & 2.86 & $C_{M E S}$ & $0.000131 \mathrm{~F}$ \\
$Q_{E S}$ & 5.05 & $L_{C E S}$ & $0.000309 \mathrm{H}$ \\
$Q_{T S}$ & 1.83 & $R_{A T}$ & $7.07 \mathrm{e} 6 \mathrm{~N} . \mathrm{s} / \mathrm{m}^{5}$ \\
$V_{A S}$ & $0.002213 \mathrm{~L}$ & $R_{M T}$ & $0.01 \mathrm{~N} . \mathrm{s} / \mathrm{m}^{5}$ \\
$C_{M S}$ & $0.001066 \mathrm{~mm} / \mathrm{N}$ & $M_{M D}$ & $3.63 \mathrm{e}-5 \mathrm{~kg}$ \\
$M_{M S}$ & $3.79 \mathrm{e}-5 \mathrm{~kg}$ & $L_{e}$ & $2.62 \mathrm{e}-5 \mathrm{H}$ \\
$R_{M S}$ & $0.066 \mathrm{~N} . \mathrm{s} / \mathrm{m}$ & $R_{E}^{\prime}$ & $61.72 \mathrm{~m} \mathrm{~m}^{2}$ \\
\hline \hline
\end{tabular}

diaphragm and the $X, Y$-displacements of the voice-coil are set to zero. The fundamental resonance frequency calculated by the modal analysis is $803 \mathrm{~Hz}$ and the associated mode shape is shown in Fig. 5(a). The fundamental mode known as the piston mode can be used to "fine-tune" FEA parameters to match the measured data. The measured result of the fundamental resonance frequency is $792 \mathrm{~Hz}$ which is about $1.4 \%$ lower than the FEA prediction. Fig. 5(b) shows another higher order mode at $18978 \mathrm{~Hz}$, where major motion takes place at the center circular portion inside the voice-coil bobbing, while the outer ring of the diaphragm is almost motionless. Due to this nature, 


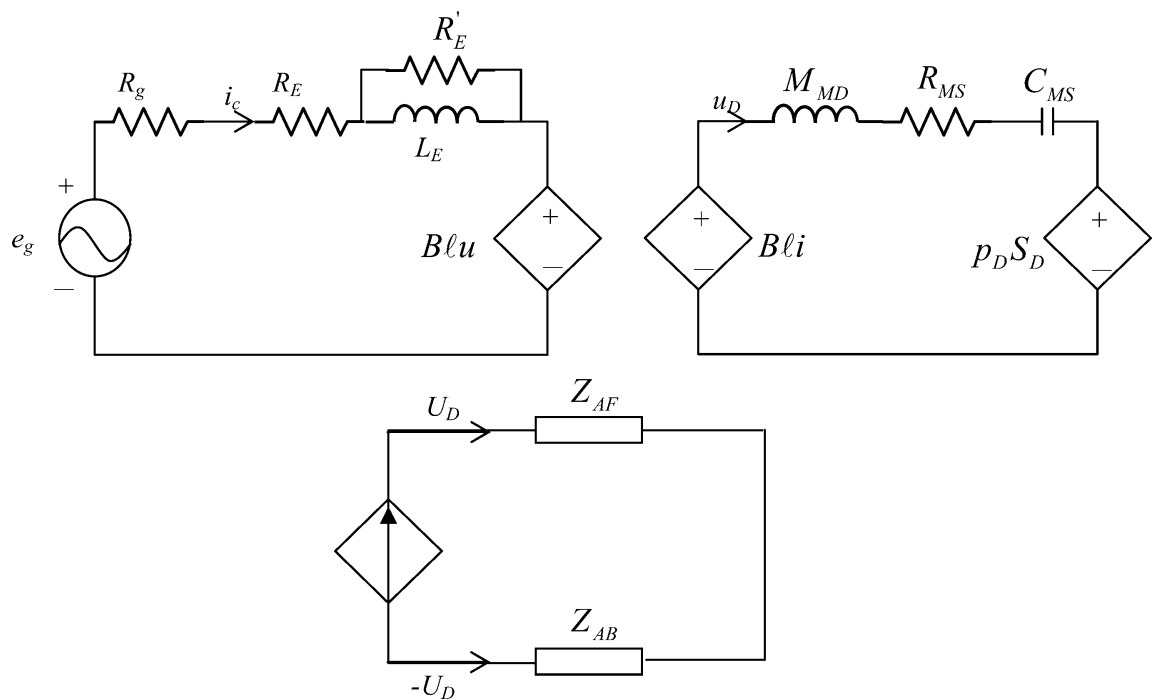

Fig. 2. EMA analogous circuit of the moving-coil dynamic microspeaker.

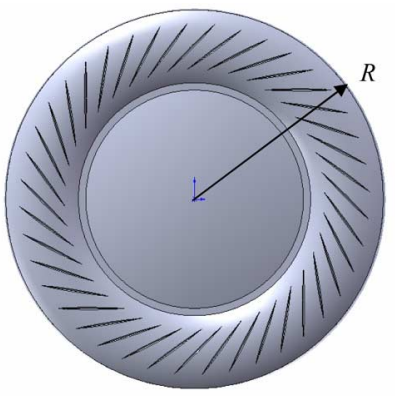

(a)

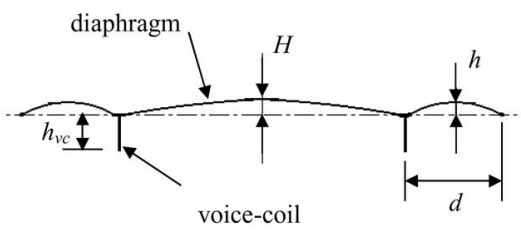

(b)

Fig. 3. Schematic diagrams of the diaphragm and voice-coil of the microspeaker. (a) Top view. (b) Cross section.

TABLE II

DIMENSIONS OF THE DIAPHRAGM AND VOICE-COIL ASSEMBLY OF THE MICROSPEAKER

\begin{tabular}{cc}
\hline Parameters & Value \\
Radius of diaphragm, $R$ & $7.8 \mathrm{~mm}$ \\
Thickness of diaphragm, $t$ & $0.029 \mathrm{~mm}$ \\
Height of inner arc, $H$ & $0.5 \mathrm{~mm}$ \\
Height of outer arc, $h$ & $0.4 \mathrm{~mm}$ \\
Bandwidth of outer arc, $d$ & $3 \mathrm{~mm}$ \\
Height of voice-coil, $h_{v c}$ & $10 \mathrm{~mm}$ \\
\hline \hline
\end{tabular}

we call it the second piston mode. The SPL response shows a peculiar boost above the second piston mode, as will be seen in the experimental results.

In order to fit the aforementioned FEA model into the analogous circuit of the microspeaker system, the dynamics of the
TABLE III

Material Properties of THE DiAPHRAGM AND VOICE-COIL ASSEMBLY

\begin{tabular}{ccc}
\hline Parameters & Diaphragm & Voice-coil \\
\hline Young's modulus $\left(\mathrm{N} / \mathrm{m}^{2}\right)$ & $8.8 \mathrm{e} 9$ & $2.4 \mathrm{e} 11$ \\
Poisson's ratio & 0.3 & 0.33 \\
Density $\left(\mathrm{kg} / \mathrm{m}^{3}\right)$ & 1600 & 8900 \\
Thickness $(\mathrm{m})$ & 0.029 & 0.2 \\
\hline \hline
\end{tabular}

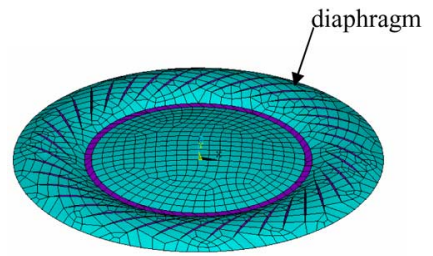

(a)

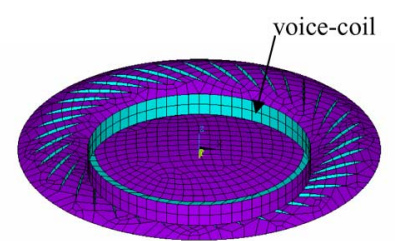

(b)

Fig. 4. Finite-element mesh of the diaphragm-voice coil assembly. (a) Front view. (b) Rear view.

FEA model has to be adapted into a lumped parameter model next. To begin with, the short-circuit mechanical impedance $\left(Z_{\mathrm{ms}}\right)$ defined in the following expression is calculated using the FEA harmonic analysis:

$$
Z_{\mathrm{ms}}=\frac{f}{\bar{u}}
$$

where $\bar{u}$ denotes the mean velocity of the diaphragm and $f$ is the excitation force delivered by the voice-coil unit

$$
f=B l i .
$$




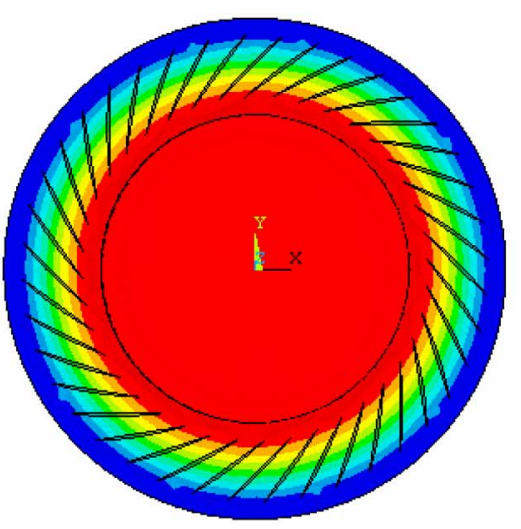

(a)

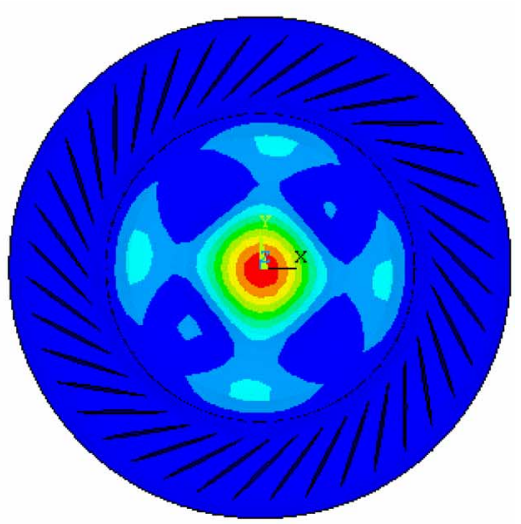

(b)

Fig. 5. Mode shapes of the diaphragm-voice coil assembly obtained using the FEA. (a) The first piston mode. (b) The second piston mode.

To calculate $Z_{\mathrm{ms}}$, the excitation force is set to be $0.13 \mathrm{~N}$. The damping ratio is assumed to be 0.16 and 0.07 for $20-4000 \mathrm{~Hz}$ and for $4 \mathrm{k}-20 \mathrm{kHz}$, respectively. The complex displacements calculated using the FEA harmonic analysis are then converted into the average velocity $(\bar{u})$. Using (2), the mechanical impedance of the diaphragm-voice coil assembly $Z_{\mathrm{ms}}$ can be calculated, as shown in Fig. 6. As a result, the dynamics of the flexible diaphragm is represented by a frequency-dependent impedance element and is readily integrated into the analogous circuit.

\section{Modeling of the Acoustical Enclosure}

The acoustical loading mainly results from the front cap, the cavity between the front cap and the diaphragm, and the acoustic radiation, as shown in Fig. 1(a). The front cap has a circular hole at the center whose acoustical impedance can be written in the acoustical domain as [9]

$$
\begin{aligned}
Z_{\mathrm{AP}} & =R_{\mathrm{AP}}+j \omega M_{\mathrm{AP}} \\
R_{\mathrm{AP}} & =\frac{\rho_{0}}{\pi a_{p}^{2}} \sqrt{2 \omega \mu}\left(\frac{t_{c}}{a_{p}}+2\right) \\
M_{\mathrm{AP}} & =\frac{\rho_{0} t_{c}}{\pi a_{p}^{2}}
\end{aligned}
$$

where $\rho_{0}$ is the density of air, $\mu$ is the kinematic coefficient of viscosity $\left(\mu=1.56 \times 10^{-5} \mathrm{~m}^{2} / \mathrm{s}\right), a_{p}$ is the radius of the hole,

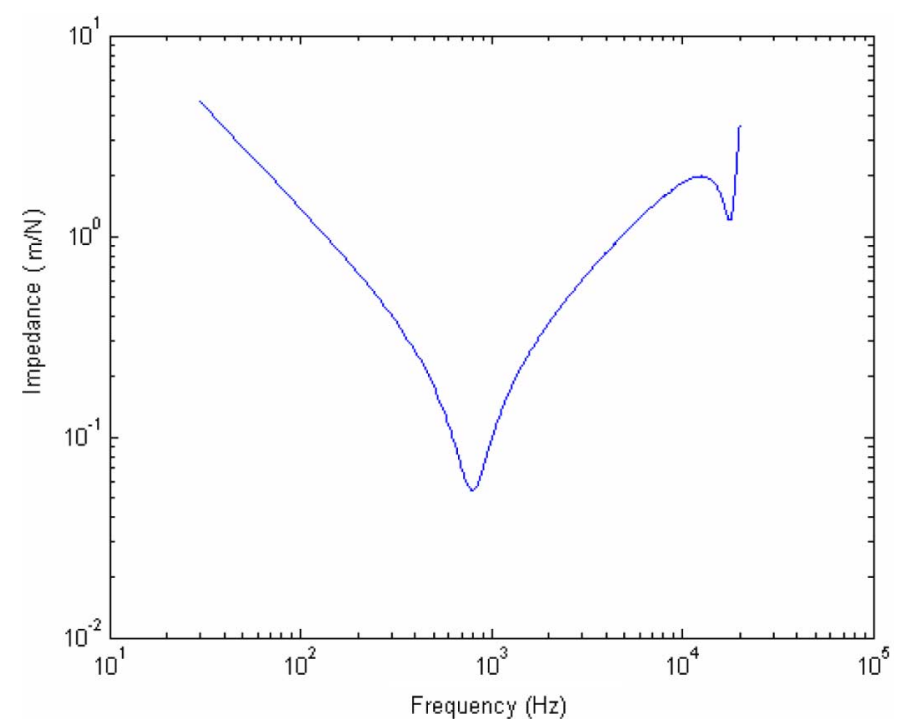

Fig. 6. Short-circuit mechanical impedance of the diaphragm-voice coil assembly.

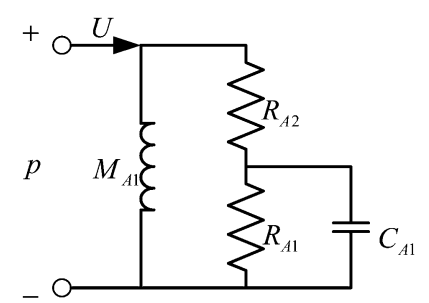

Fig. 7. Analogous circuit of the radiation impedance.

and $t_{c}$ is the thickness of the front cap. The cavity can be model as the acoustic compliance.

$$
C_{A}=\frac{V}{\rho_{0} c^{2}}
$$

where $V$ is the volume of cavity and $c$ is the speed of sound.

The analogous circuit of the radiation impedance for a baffled piston is shown in Fig. 7, with parameters given by [9]

$$
\begin{aligned}
M_{A 1} & =\frac{8 \rho_{0}}{3 \pi^{2} a} \\
R_{A 1} & =\frac{0.4410 \rho_{0} c}{\pi a^{2}} \\
R_{A 2} & =\frac{\rho_{0} c}{\pi a^{2}} \\
C_{A 1} & =\frac{5.94 a^{3}}{\rho_{0} c^{2}} .
\end{aligned}
$$

Fig. 8 shows the analogous circuit of the microspeaker in the acoustical domain.

\section{Frequency Response Simulation}

Loop equations can be written for the preceding FEA-lumped parameter circuit of the microspeaker as follows [21]:

$$
\left[\begin{array}{ccc}
Z_{E} & B l & 0 \\
B l & -Z_{\mathrm{ms}} & -p_{D} \\
0 & -S_{D} Z_{A} & p_{D}
\end{array}\right]\left[\begin{array}{c}
i \\
\bar{u} \\
S_{D}
\end{array}\right]=\left[\begin{array}{c}
e_{g} \\
0 \\
0
\end{array}\right]
$$




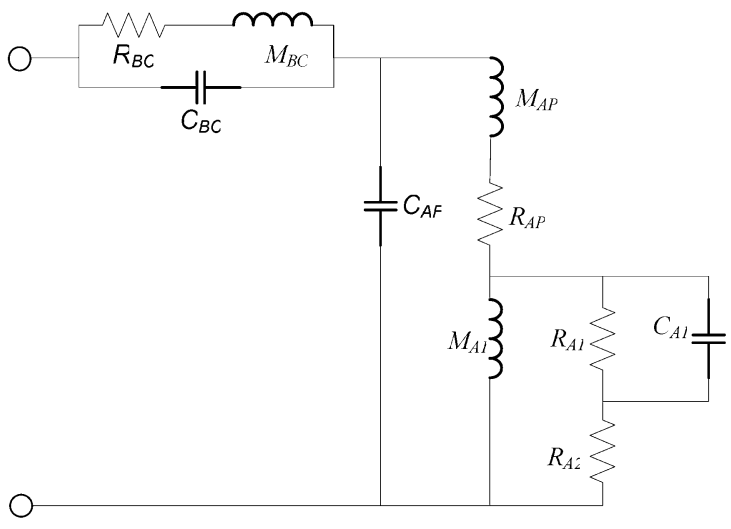

Fig. 8. Analogous circuit for modeling the acoustical enclosure of the microspeaker.

where $i$ is the current, $\bar{u}$ is the mean velocity of the diaphragm, $S_{D}$ is the effective area of the diaphragm, $e_{g}$ is the driving voltage, $s=j \omega$ is the Laplace variable, and

$$
\begin{aligned}
Z_{E} & =R_{E}+\left(R_{E}^{\prime} \| L_{E} s\right) A \\
Z_{A} & =\left[\left(Z_{R}+M_{\mathrm{AP}} s+R_{\mathrm{AP}}\right) \| \frac{1}{C_{\mathrm{AF}} s}\right]+Z_{\mathrm{BC}} \\
Z_{R} & =\left(\frac{1}{C_{A 1} s} \| R_{A 1}+R_{A 2}\right) \| M_{A 1} s \\
Z_{\mathrm{BC}} & =\left(R_{\mathrm{BC}}+M_{\mathrm{BC}} s\right) \| \frac{1}{C_{\mathrm{BC}} s}
\end{aligned}
$$

The symbol || denotes parallel connection of circuit. The loop equations can be solved for the current and velocity of the diaphragm for each frequency. From the current and velocity, the electrical impedance and the on-axis SPL responses of the microspeaker can be simulated.

\section{Design Optimization OF THE Diaphragm PATtERn}

On the basis of the preceding simulation model, optimization is carried out to determine diaphragm parameters.

\section{A. Simulation and Measurement of Frequency Responses}

Simulations and experiments are undertaken in this paper to validate the aforementioned integrated microspeaker model. The frequency response from $20 \mathrm{~Hz}$ to $20 \mathrm{kHz}$ of the microspeaker is measured using a 2 Vrms sweep sine input. Fig. 9(a) shows the experimental arrangement for measuring voice-coil impedance (with symbols defined in the figure):

$$
Z_{\mathrm{vc}}=\frac{e_{s}}{e_{g}-e_{s}} R
$$

Fig. 9(b) shows the experimental arrangement for measuring the on-axis SPL response by using a microphone positioned at $5 \mathrm{~cm}$ away from the microspeaker.

Next, simulation of the diaphragm response was carried out using the integrated FEA-lumped parameter model mentioned

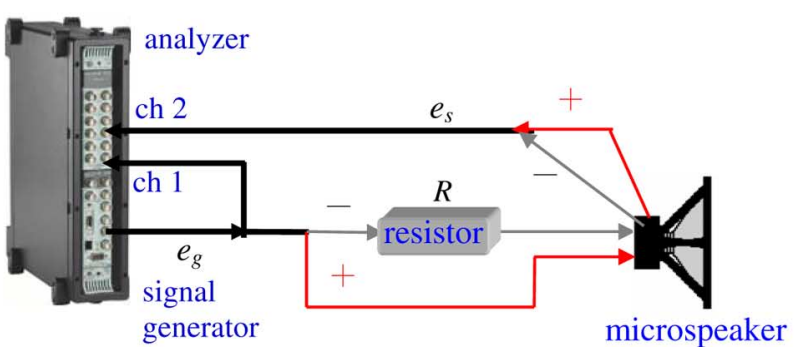

(a)

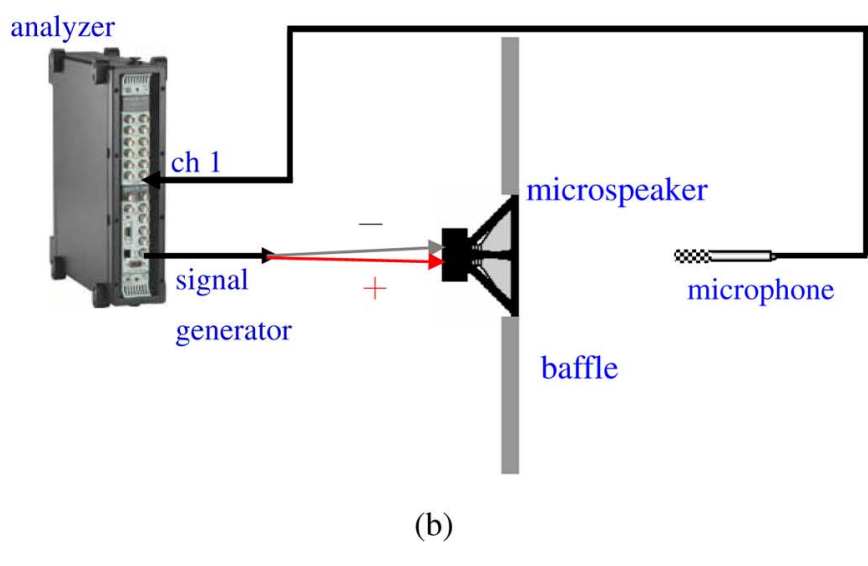

Fig. 9. Experimental arrangement for the microspeaker measurement. (a) Setup for voice-coil impedance measurement. (b) Setup for on-axis SPL response.

above. Fig. 10(a) and (b) compares the voice-coil impedance and the on-axis SPL obtained from the simulation and the experiment, respectively. It can be observed that response predicted by conventional lumped parameter model is in good agreement with the measurement in low frequencies. In high frequencies, the conventional approach fails to capture the response due to the flexural modes of the diaphragm. However, the response simulated by the integrated FEA-lumped parameter model matches the measured response quite well.

\section{B. Diaphragm Optimization Using the Taguchi Method}

As mentioned previously, the diaphragm pattern has major impact on the microspeaker response. To pinpoint the optimal pattern design, the Taguchi method and sensitivity analysis are exploited in this study. The Taguchi method [18] is very useful for experimental design, particularly for problems with finite number of discrete levels of design factors and thus reduction of the number of experiments is highly desired. In the following, the dimensions of diaphragm-voice coil assembly of microspeakers will be optimized by using the Taguchi method. Table IV shows the $L_{9}\left(3^{4}\right)$ orthogonal array to be used in the Taguchi procedure. Here, nine observations and four factors are involved. The factors, each discretized into three levels, include the height of inner $\operatorname{arc}(H)$, the height of outer $\operatorname{arc}(h)$, 


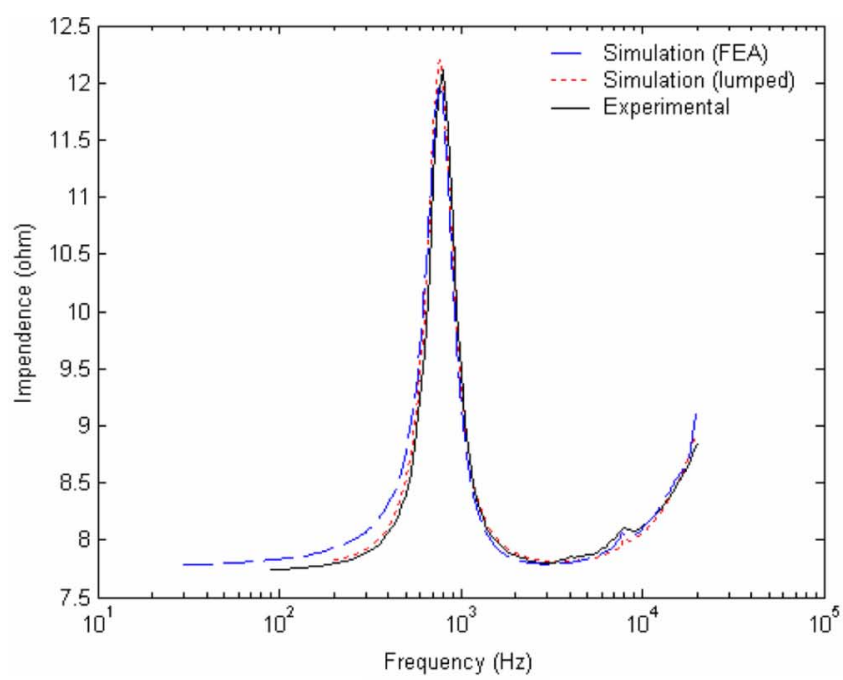

(a)

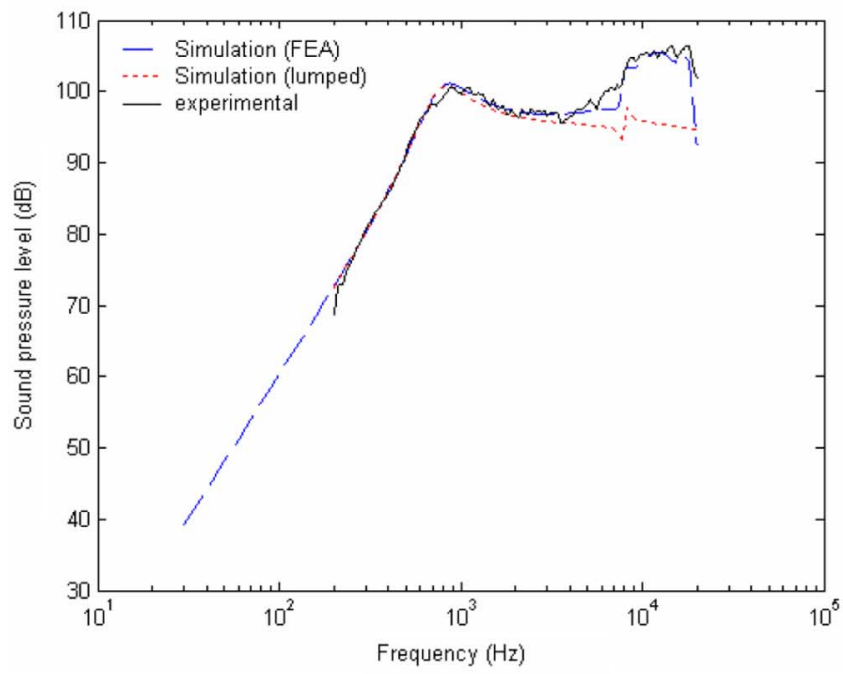

(b)

Fig. 10. Simulated and measured frequency responses of the microspeaker. (a) Voice-coil impedance. (b) On-axis SPL response.

the bandwidth of outer arc $(d)$, and the thickness of diaphragm $(t)$, as summarized in Table IV.

The following procedure aims to find the optimal parameters for the microspeaker diaphragm design according to the cost function:

$$
\begin{gathered}
f_{c}=-\frac{\Delta f_{0}}{f_{0}} \times w_{1}+\frac{\Delta f_{1}}{f_{1}} \times w_{2} \\
+\frac{\Delta \overline{\mathrm{SPL}}}{\overline{\mathrm{SPL}}} \times w_{3}-\frac{\Delta \mathrm{STD}}{\mathrm{STD}} \times w_{4}
\end{gathered}
$$

where $f_{0}$ is the lower cutoff frequency of microspeaker, $f_{1}$ is the upper cutoff frequency of microspeaker, $\overline{\mathrm{SPL}}$ denotes the mean SPL in the piston bandwidth (defined in Fig. 11), and STD denotes the standard deviation of SPL in the piston bandwidth that serves as a flatness measure

$$
\mathrm{STD}=\sqrt{\frac{1}{n-1} \sum_{i=1}^{n}\left(\mathrm{SPL}_{i}-\overline{\mathrm{SPL}}\right)^{2}}
$$

TABLE IV

$L_{9}\left(3^{4}\right)$ ORTHOGONAL ARRAY OF THE TAGUCHI METHOD

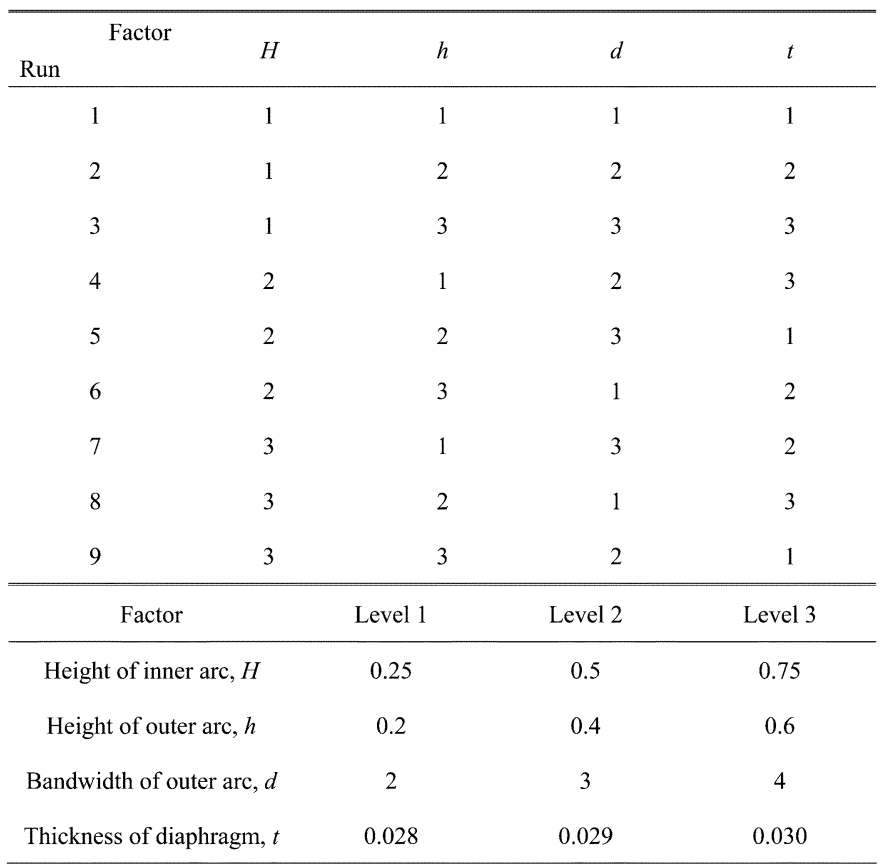

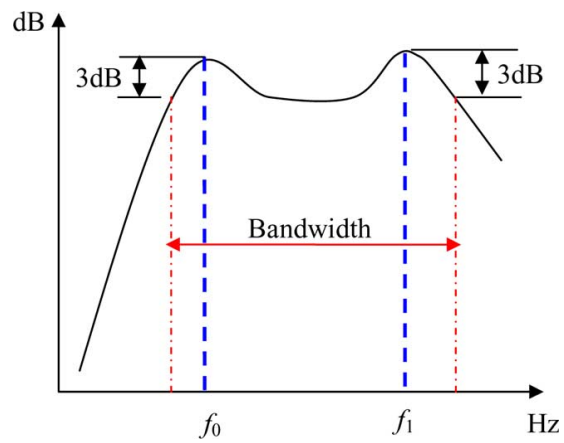

Fig. 11. Definitions of performance parameters of the SPL response.

TABLE V

FOUR WeIGHTING SCHEMES FOR THE COST FunCTION

\begin{tabular}{ccccc}
\hline Weighting & Scheme 1 & Scheme 2 & Scheme 3 & Scheme 4 \\
\hline$w_{1}$ & 0.25 & 0.35 & 0.25 & $0.8 / 3$ \\
$w_{2}$ & 0.25 & 0.15 & 0.15 & $0.8 / 3$ \\
$w_{3}$ & 0.25 & 0.25 & 0.35 & $0.8 / 3$ \\
$w_{4}$ & 0.25 & 0.25 & 0.25 & 0.2 \\
\hline
\end{tabular}

where $n$ is the number of frequency components of SPL in the band and $\mathrm{SPL}_{i}$ is the $i$ th SPL in the band. The symbol $\Delta$ signifies the difference of the performance parameters between the original design and the Taguchi design, e.g., $\Delta f_{0}=$ $f_{0, \text { Taguchi }}-f_{0, \text { original }}, w_{j}, j=1 \sim 4$, is the weight for the performance parameter $i$. In order to accommodate more design objectives, we consider using four kinds of weighting schemes, and summarized in Table V. In scheme 1, the weights for the performance parameters are equal. Larger weights are used to emphasize the lower cutoff frequency and the mean of SPL in evaluating schemes 2 and 3, respectively. In the weighting 


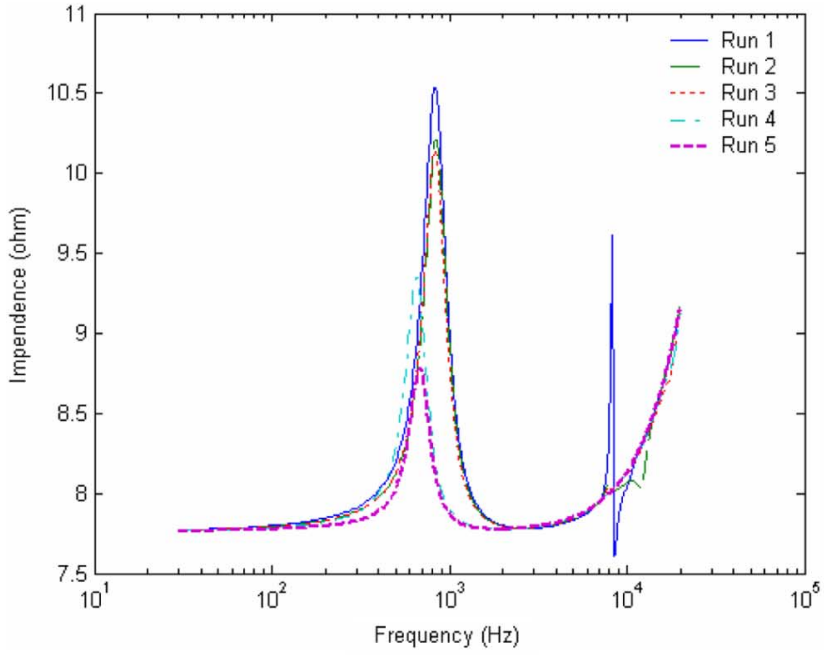

(a)

(b)

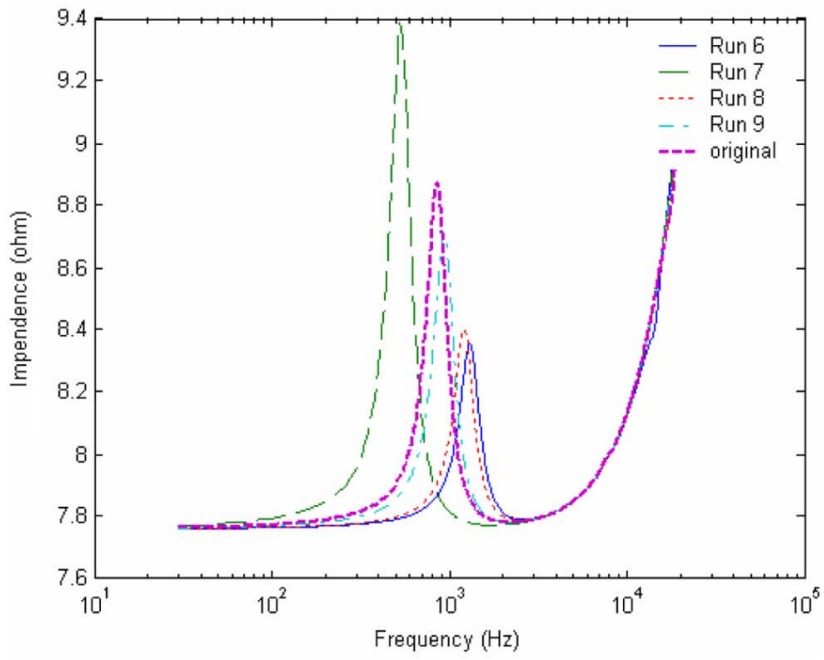

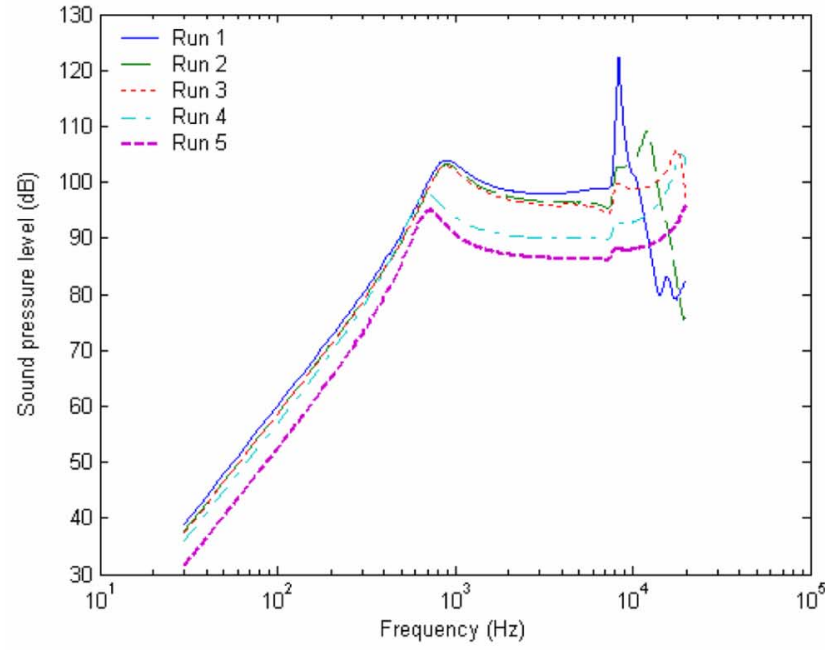

(c)

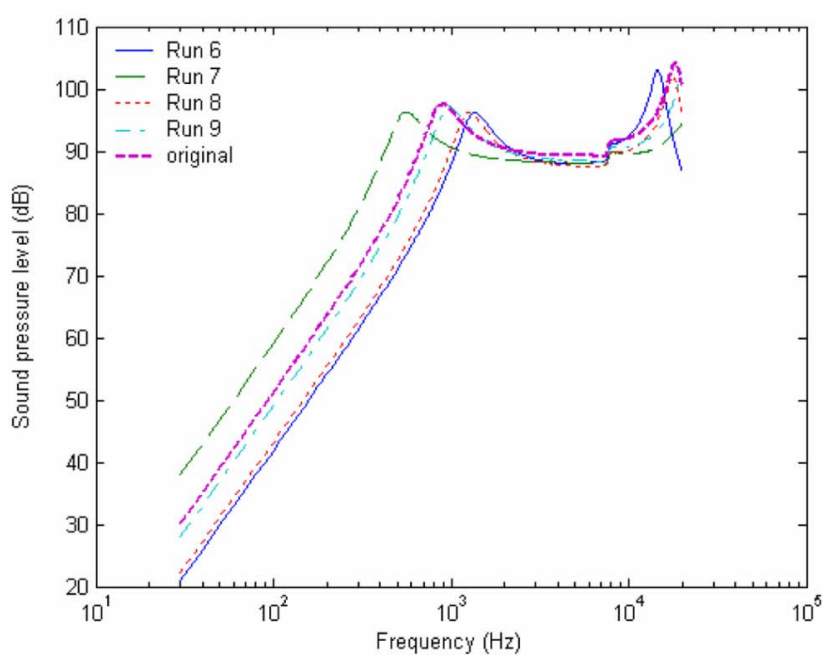

(d)

Fig. 12. Simulated voice-coil impedance and the on-axis SPL response obtained in the Taguchi analysis. (a) Voice-coil impedance for Runs 1-5. (b) Voice-coil impedance for Runs 6-9. (c) On-axis SPL for Runs 1-5. (d) On-axis SPL for Runs 6-9.

scheme 4, however, more emphasis is placed on the lower cutoff frequency, the upper cutoff frequency and the mean of SPL than the standard deviation of SPL.

Fig. 12(a)-(d) shows the simulated voice-coil impedance and the SPL response of Run 1 to 9 in the $L_{9}\left(3^{4}\right)$ orthogonal array. The values of calculated cost function for all weighting schemes are summarized in Table VI. The cost function of Run 7 has attained the highest value among all schemes. In Run 7, the lower cutoff frequency is reduced to $567.3 \mathrm{~Hz}$, the upper resonance frequency is increased to $20 \mathrm{kHz}$, the SPL of the resonance frequency is increased to $84.8 \mathrm{~dB}$, and the standard deviation is $1.88 \mathrm{~dB}$. The optimal design result indicates that the height of inner $\operatorname{arc}(H)$ and the width of the outer arc $(d)$ should be as large as possible, and the height of outer arc $(h)$ should be as small as possible, which will maximize the cost functions.

\section{Sensitivity Analysis of Corrugation}

Sensitivity analysis of diaphragm corrugation is undertaken to examine the effect of corrugation number on the micros-
TABLE VI

VALUES OF THE COST FUNCTION IN THE TAGUCHI ANALYSIS FOR NINE RUNS AND FouR WEIGHTING SCHEMES

\begin{tabular}{ccccc}
\hline Run & Scheme 1 & Scheme 2 & Scheme 3 & Scheme 4 \\
\hline 1 & -0.0610 & -0.0052 & 0.0025 & -0.0279 \\
2 & -0.0080 & 0.0242 & 0.0322 & 0.0136 \\
3 & 0.1029 & 0.1068 & 0.1119 & 0.1116 \\
4 & 0.0634 & 0.0836 & 0.0611 & 0.0657 \\
5 & 0.1714 & 0.1835 & 0.1575 & 0.1770 \\
6 & -0.1731 & -0.2035 & -0.1541 & -0.1712 \\
7 & $\mathbf{0 . 2 5 0 6}$ & $\mathbf{0 . 2 7 9 2}$ & $\mathbf{0 . 2 3 8 1}$ & $\mathbf{0 . 2 6 1 5}$ \\
8 & -0.0970 & -0.1349 & -0.0960 & -0.1016 \\
9 & 0.0533 & 0.0369 & 0.0430 & 0.0510 \\
\hline \hline
\end{tabular}

peaker performance. The analysis is based on the optimal diaphragm dimensions obtained in Run 7 of the preceding Taguchi 


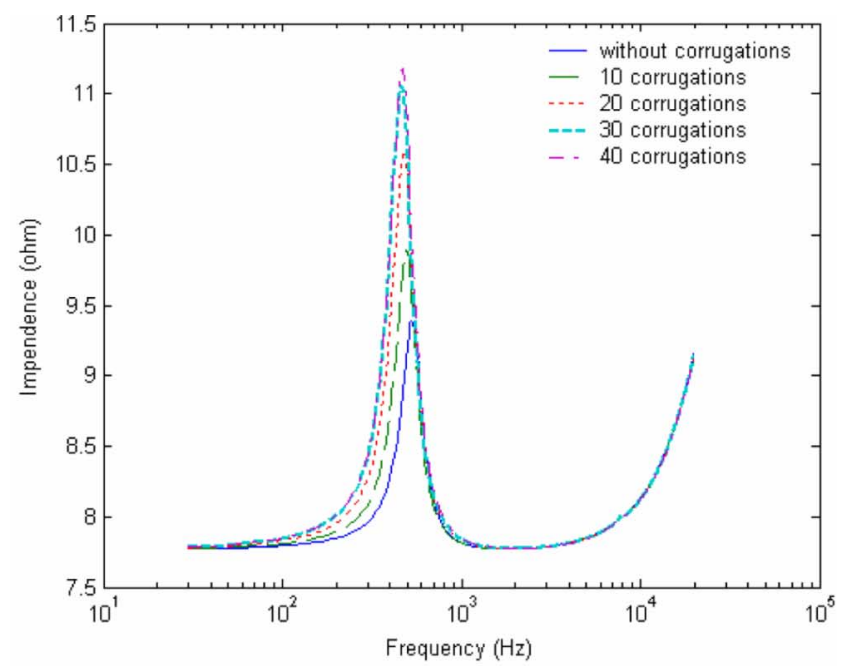

(a)

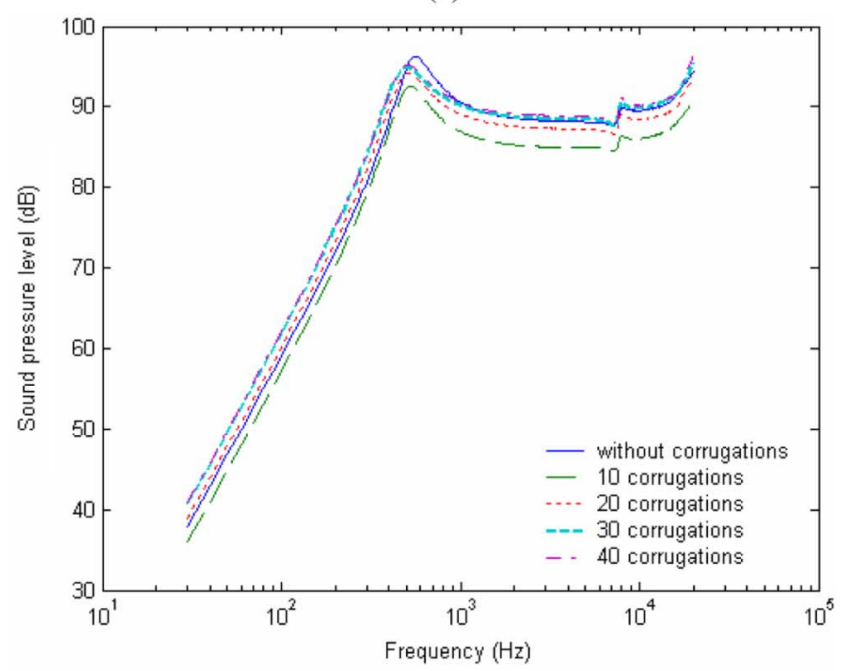

(b)

Fig. 13. Simulated frequency responses of Run 7 for different number of corrugation. (a) Voice-coil impedance. (b) On-axis SPL response.

procedure. The simulated voice-coil impedance and SPL response of Run 7 for different corrugation numbers are shown in Fig. 13(a)-(b). The values of performance indices and the resulting cost function for different corrugation numbers are summarized in Table VII. It can be observed that corrugation tends to reduce the fundamental resonance frequency, but the relation is not linear. Further, corrugation tends to increase the mean and the standard deviation of the SPL response. In another word, increasing the number of corrugations will decrease the flatness of SPL during the effective frequency range. Nevertheless, the corrugation does not seem to affect the upper cutoff frequency significantly.

The values of cost function in relation to the corrugation number for different weighting schemes are also summarized in Table VII. The values of cost function are derived from the result of Run 7 of the Taguchi method. The results reveal that the optimal corrugation number is 30 , in which the cost function is within $0.0493-0.0616$ for the four weighting schemes.
TABLE VII

Calculated Performance Parameters of Sensitivity Analysis AND THE ASSOCIATED COST FUNCTION

\begin{tabular}{ccccc}
\hline Corrugation & $f_{0}(\mathrm{~Hz})$ & $f_{1}(\mathrm{~Hz})$ & $\overline{S P L}(\mathrm{~dB})$ & $S T D$ \\
\hline 0 & 567.25 & 20000 & 84.8 & 1.88 \\
10 & 527.45 & 20000 & 86.4 & 1.80 \\
20 & 507.55 & 20000 & 88.7 & 1.81 \\
30 & 497.60 & 20000 & 90.0 & 1.85 \\
40 & 507.55 & 20000 & 90.3 & 1.88 \\
\hline \hline Corrugation & Scheme 1 & Scheme 2 & Scheme 3 & Scheme 4 \\
\hline 10 & 0.0324 & 0.0394 & 0.0343 & 0.0346 \\
20 & 0.0465 & 0.0570 & 0.0511 & 0.0496 \\
$\mathbf{3 0}$ & $\mathbf{0 . 0 4 9 3}$ & $\mathbf{0 . 0 6 1 6}$ & $\mathbf{0 . 0 5 5 4}$ & $\mathbf{0 . 0 5 2 6}$ \\
40 & 0.0418 & 0.0523 & 0.0483 & 0.0446 \\
\hline
\end{tabular}

\section{CONCLUSION}

An FEA-lumped parameter model has been presented for electroacoustical simulation of microspeakers. The mechanical impedance obtained from the FEA model of the diaphragm-voice coil assembly is incorporated into the lumped parameter model of the microspeaker system. The integrated EMA model provides better prediction for the voice-coil impedance and the SPL response at high frequencies than the conventional lumped parameter approach that neglects the higher-order flexural modes of the diaphragm.

On the basis of the proposed model, the dimensions of diaphragm are optimized with the aid of the Taguchi method. Using the results of the Taguchi method as a starting point, the optimal number of diaphragm corrugation is determined using sensitivity analysis. According to the optimized design of Table VI, the fundamental resonance frequency has been decreased and the flatness of the SPL response has been improved over the nonoptimized design.

In the future research, the physical parameters and the response parameters of the microspeaker will be correlated by using regression analysis or neural-fuzzy network. On the platform of this simulation model, more efficient optimization techniques shall be developed to determine design parameters of microspeakers.

\section{ACKNOWLEDGMENT}

This work was supported by the National Science Council in Taiwan under Project NSC 95-2221-E-009-009-MY2.

\section{REFERENCES}

[1] I. Chun, P. A. Nelson, and J. T. Kim, "Numerical models of miniature loudspeakers," in 32nd Int. Congr. Exposition Noise Control Eng., Jeju Island, Korea, Aug. 2003.

[2] S. J. Oh, H. R. Lee, S. W. Yoon, and J. S. Park, "Study of the acoustical properties as a function of back cavity for loudspeaker," in 32nd Int. Congr. Exposition Noise Control Eng., Jeju Island, Korea, Aug. 2003. 
[3] C. H. Choi and H. S. Yoon, "Acoustic and vibration characteristics of a micro speaker through the electro-magnetic field analysis," in 32nd Int. Congr. Exposition Noise Control Eng., Jeju Island, Korea, Aug. 2003.

[4] S. H. Lee, J. H. Kim, J. T. Kim, O. S. Kwon, and C. H. Choi, "Development of the simulation program to analyze acoustic characteristics of a miniature type loudspeaker," in 32nd Int. Congr. Exposition Noise Control Eng., Jeju Island, Korea, Aug. 2003.

[5] A. Bright, "Simplified loudspeaker distortion compensation by DSP," in 23rd AES Int. Conf., Copenhagen, Denmark, May 2003.

[6] M. R. Bai and R. L. Chen, "Optimal design of loudspeaker systems based on sequential quadratic programming (SQP)," J. Audio Eng. Soc., vol. 55, no. 1/2, pp. 44-54, 2007.

[7] H. Olson, Acoustical Engineering. Philadelphia, PA: Van Nostrand, 1957, Reprinted by Professional Audio Journals 1991.

[8] L. L. Beranek, Acoustics. Woodbury, NY: Acoustical Society of America, 1996.

[9] W. M. Leach Jr., Introduction to Electroacoustics and Audio Amplifier Design. Dubuque, IA: Kendall-Hunt, 2003.

[10] N. Thiele and R. Small, "Audio engineering society," in AES Loudspeaker Anthologies 1978, 1984, New York, 1996, vol. 1-3.

[11] M. R. Bai and J. Liao, "Acoustic analysis and design of miniature loudspeakers for mobile phones," Audio Eng. Soc., vol. 53, no. 11, pp. 1061-1076, 2005.

[12] S. M. Hwang, G. Y. Hwang, J. H. Kwon, H. J. Lee, and B. S. Kang, "Performance comparison between circular and elliptical type microspeakers for cellular phones," IEEE Trans. Magn., vol. 39, no. 5, pp. 3256-3258, Sep. 2003.

[13] J. H. Kwon, S. M. Hwang, and K. S. Kim, "Development of slim rectangular microspeaker used for minimultimedia phones," IEEE Trans. Magn., vol. 43, no. 6, pp. 2704-2706, Jun. 2007.
[14] M. Opitz and R. Barnert, "Modern development tools for dynamic transducers," in AES 111th Convention, New York, , Sep. 2001.

[15] J. H. Kwon and S. M. Hwang, "Analysis of acoustic characteristics according to design parameter of diaphragm," J. Mater. Process. Tech. vol. 187-188, pp. 442-446, 2007.

[16] P. C. P. Chao, C. W. Chiu, and H. P. Yuan, "Magneto-electrodynamical modeling and design of a microspeaker used for mobile phones with considerations of diaphragm corrugation and air closures," IEEE Trans. Magn., vol. 43, no. 6, pp. 2585-2587, Jun. 2007.

[17] C. A. Desoer and E. S. Kuh, Basic Circuit Theory. New York: McGraw-Hill, 1969.

[18] C. R. Hicks and K. V. Turner, Fundamental Concepts in the Design of Experiments. Oxford, , U.K.: Oxford Univ. Press, 1999.

[19] M. R. Bai and Y. Lu, "Optimal implementation of miniature piezoelectric panel speakers using the Taguchi method and genetic algorithm," J. Vib. Acoust., vol. 126, pp. 359-369, 2004.

[20] Swanson Analysis Systems, ANSYS User's Manual. Philadelphia, PA, 2005.

[21] C. A. Desoer and E. S. Kuh, Basic Circuit Theory. New York: McGraw-Hill, 1969.

Manuscript received February 4, 2008; revised April 7, 2008. Corresponding author: M. R. Bai (e-mail: msbai@mail.nctu.edu.tw). 